\title{
GENERATING FUNCTIONS ON COVERING GROUPS
}

\author{
DAVID GINZBURG
}

\begin{abstract}
In this paper we prove Conjecture 1.2 in B-F. This enables us to establish the meromorphic continuation of the standard partial $L$ function $L^{S}\left(s, \pi^{(n)}\right)$. Here, $\pi^{(n)}$ is a genuine irreducible cuspidal representation of the group $G L_{r}^{(n)}(\mathbf{A})$.
\end{abstract}

\section{INTRODUCTION}

Let $\mathbf{A}$ denote the ring of adeles of a global field $F$. Assume that $F$ contains the $n$ roots of unity. Let $G L_{r}^{(n)}(\mathbf{A})$ denote the $n$ fold metaplectic cover of the group $G L_{r}(\mathbf{A})$. Let $\pi^{(n)}$ denote a genuine irreducible cuspidal representation of $G L_{r}^{(n)}(\mathbf{A})$. As explained in [B-F], to this representation one can attach the partial standard $L$ function, denoted by $L^{S}\left(s, \pi^{(n)}\right)$.

In $[\mathrm{B}-\mathrm{F}]$ the authors introduced a global integral to study this $L$ function. Their idea was to take the well known Rankin-Seleberg convolution of two cuspidal representation of $G L_{r}(\mathbf{A})$ and $G L_{n}(\mathbf{A})$. For the constructions of these Rankin-Selberg integrals, and the motivations for the adopted construction in the covering groups, we refer to $[\mathrm{B}-\mathrm{F}]$. Then, they replaced the cuspidal representation of $G L_{n}(\mathbf{A})$ by the Theta representation $\Theta_{n}^{(n)}$. This last representation was constructed in [K-P]. For example, if $r>n$, the construction suggested in $[\mathrm{B}-\mathrm{F}]$ is given by

$$
\int_{G L_{r}(F) \backslash G L_{r}(\mathbf{A}) V_{r, n}(F) \backslash V_{r, n}(\mathbf{A})} \phi\left(v\left(\begin{array}{ll}
g & \\
& I_{r-n}
\end{array}\right)\right) \overline{\theta(g)} \psi_{V_{r, n}}(v)|\operatorname{det} g|^{s-\frac{r-n}{2}} d v d g
$$

Here $\phi$ is a vector in the space of $\pi^{(n)}$, and $\theta$ is a vector in the space of $\Theta_{n}^{(n)}$. The group $V_{r, n}$, and the character $\psi_{V_{r, n}}$ are defined in the last section. In fact in $\mathrm{B}-\mathrm{F}$ ] the authors concentrated on the case when $r<n$, but up to some modifications as explained in [B-F] Section 2, the idea is the same.

A straight-forward unfolding, implies that for $\operatorname{Re}(s)$ large, integral (1) is equal to

$$
\int_{V_{r}(\mathbf{A}) \backslash G L_{r}(\mathbf{A})} W_{\phi}\left(\begin{array}{ll}
g & \\
& I_{r-n}
\end{array}\right) \overline{W_{\theta}(g)}|\operatorname{det} g|^{s-\frac{r-n}{2}} d g
$$

Here $W_{\phi}$ denotes the Whittaker coefficient of $\phi$, and similarly we define $W_{\theta}$.

Since the Whittaker coefficient $W_{\phi}$ is not factorizable, it is not obvious that the above integral represents an Euler product. To show that it does, one needs to apply the method referred to as the New Way which was developed in [PS-R]. See [B-F] for a discussion and references for this method.

2010 Mathematics Subject Classification. Primary 11F70; Secondary 11F55, 11F66.

The author is partly supported by the Israel Science Foundation grant number 259/14. 
In our context, to deduce that integral (2) is Eulerian one can proceed in two steps. The first step is to find a generating function for the unramified local $L$ function $L\left(s, \pi^{(n)}\right)$. See B-F page 5 for its definition. In [B-F], such a function was introduced, and was denoted by $\widetilde{\Delta}_{s}$. The second step is to use this function to compute a local integral which is obtained from integral (2), and to show that this local integral is independent of the choice of the local Whittaker function attached to the local component of $\phi$. To do that one needs to compute the Whittaker function of $\widetilde{\Delta}_{s}$. This is done in $[\mathrm{B}-\mathrm{F}]$ for the cases $r=n=2,3$. The general case is conjectured in $\mathrm{B}-\mathrm{F}$ Conjecture 1.2.

In this paper we prove Conjecture 1.2 of $[\mathrm{B}-\mathrm{F}]$ in complete generality. To do this we give a different realization for the function $\widetilde{\Delta}_{s}$. This realization makes the proof of the stated conjecture relatively simple. The new realization is described in Section 2 and is given by a certain unique functional defined on the local Theta representation $\Theta_{n r}^{(n)}$ defined on the group $G L_{n r}^{(n)}$. We then use this functional to define a function on the group $G L_{n r}^{(n)}$, which we denote by $W_{n r}^{(n)}(h)$. Here $h \in G L_{n r}^{(n)}$. Restricting to the group $G L_{r}^{(n)}$ we obtain a function on that group which we use to give the new expression for $\widetilde{\Delta}_{s}$. Thus our result contains two parts. The first is the proof that the function $W_{n r}^{(n)}(h)$ restricted to $G L_{r}^{(n)}$ is indeed the generating function for the standard $L$ function. This we do in Proposition 2. The second, and the main result of this paper, is to obtain the desired expression for the Whittaker function of the generating function. This we do in Theorem [2, which is Conjecture 1.2 in [B-F]. In both cases the computations are quite straight forward and are done by a repeated application of Lemma 1 and Corollary 1 stated and proved in Subsection 2.1.

As mentioned above, the global result and some of the computations done in [B-F] assumes that $r<n$. This is just a technical point. The authors of $[\mathrm{B}-\mathrm{F}]$ were well aware that their construction works for all $r$ and $n$. To complete their result, in the last section we give some details in the other two cases, that is the case when $r>n$ and $r=n$.

To summarize, combining [B-F] with our result we have

Theorem 1. Let $\pi^{(n)}$ denote an irreducible cuspidal representation of the group $G L_{r}^{(n)}(\mathbf{A})$. Then the partial $L$ function $L^{S}\left(s, \pi^{(n)}\right)$ has a meromorphic continuation to the whole complex plane. When $r \neq n$ this partial $L$ function is holomorphic. When $r=n$ it can have at most a simple pole at $s=1$.

As a final remark we mention that in fact we do expect that the partial $L$ function $L^{S}\left(s, \pi^{(n)}\right)$ will also be holomorphic in the case when $r=n$. From the global integral given in the last section we deduce that if this $L$ function has a simple pole at $s=1$, then $\pi^{(n)}$ will be isomorphic to $\Theta_{n}^{(n)}$. This we believe cannot happen.

\section{Generating functions}

The main references for this section are $[\mathrm{B}-\mathrm{F}]$ and $[\mathrm{K}-\mathrm{P}]$. Fix a positive integer $n>1$. Let $F$ denote a local nonarchimedean field which contains the $n$ roots of unity. Let $G L_{r}^{(n)}$ denote the metaplectic $n$ fold cover of the group $G L_{r}$. Let $\pi^{(n)}$ denote an unramified representation of $G L_{r}^{(n)}$ attached to a character $\chi$ of the torus $T_{r}$ of $G L_{r}$. This is explained in [B-F] page 5. Assuming that $\chi$ is in general position, one can attach to $\pi^{(n)}$, the local $L$ function which is 
defined as

$$
L\left(s, \pi^{(n)}\right)=\frac{1}{\prod_{i=1}^{r}\left(1-\chi_{i}^{n}(p) q^{-s}\right)}
$$

Here $p$ is a generator of the maximal ideal in the ring of integers of $F$, and $q^{-1}=|p|_{F}$. Also, $s$ is a complex variable.

In [B-F] formula (1.4) the function $\widetilde{\Delta}_{s}(h)$ is defined. This is a function of $G L_{r}^{(n)}$, and Proposition 1.1 in $[\mathrm{B}-\mathrm{F}]$ states that for $\operatorname{Re}(s)$ large,

$$
\int_{G L_{r}} \omega_{\pi^{(n)}}(h) \widetilde{\Delta}_{s}(h) d h=L\left(s, \pi^{(n)}\right)
$$

Here, $\omega_{\pi^{(n)}}$, denoted by $\sigma$ in $[\mathrm{B}-\mathrm{F}]$, is the spherical function attached to $\pi^{(n)}$. Thus $\omega_{\pi^{(n)}}$ is a $K_{r}$ bi-invariant function of $G L_{r}^{(n)}$ where $K_{r}$ is the maximal compact subgroup of $G L_{r}$ embedded in $G L_{r}^{(n)}$ as described in $[\mathrm{B}-\mathrm{F}]$. As is well known, the function $\widetilde{\Delta}_{s}(h)$ is uniquely determined by Proposition 1.1 in $[\mathrm{B}-\mathrm{F}]$. This function is referred to as the generating function for the standard $L$ function of the group $G L_{r}^{(n)}$.

We will give a different realization of the function $\widetilde{\Delta}_{s}(h)$. To do that let $\Theta_{n r}^{(n)}$ denote the local unramified Theta representation of $G L_{n r}^{(n)}$ as constructed in $[\mathrm{K}-\mathrm{P}]$. Thus, this representation is the unramified sub-representation of $\operatorname{In} d_{B_{n r}^{(n)}}^{G L_{n r}^{(n)}} \delta_{B_{n r}}^{\frac{n-1}{2 n}}$. Here, $B_{n r}$ is the Borel subgroup of $G L_{n r}$. This representation is not generic, however it still has a certain unique functional defined on it. To describe this functional, let $U_{n r}$ denote the unipotent radical of the parabolic subgroup of $G L_{n r}$ whose Levi part is $G L_{r} \times G L_{r} \times \ldots \times G L_{r}$. In term of matrices the group $U_{n r}$ consists of all matrices of the form

$$
\left(\begin{array}{ccccc}
I & X_{1,2} & X_{1,3} & \cdots & X_{1, n} \\
& I & X_{2,3} & \cdots & X_{2, n} \\
& & I & \ddots & \vdots \\
& & & \ddots & X_{n-1, n} \\
& & & & I
\end{array}\right)
$$

Here $I$ is the $r \times r$ identity matrix, and $X_{i, j} \in M a t_{r \times r}$.

Let $\psi$ denote an unramified character of $F$. Define a character $\psi_{U_{n r}}$ of $U_{n r}$ as follows. For $u \in U_{n r}$ as above, define $\psi_{U_{n r}}(u)=\psi\left(\operatorname{tr}\left(X_{1,2}+X_{2,3}+\cdots+X_{n-1, n}\right)\right)$. The stabilizer of $\psi_{U_{n r}}$ inside $G L_{r} \times G L_{r} \times \ldots \times G L_{r}$ is the group $G L_{r}^{\Delta}$ embedded diagonally. The embedding of $G L_{r}^{\Delta}$ inside $G L_{n r}$ is given by $g \mapsto \operatorname{diag}(g, g, \ldots, g)$.

Given a representation $\sigma_{n r}^{(n)}$ of $G L_{n r}^{(n)}$, we consider the space of all functionals on $\sigma_{n r}^{(n)}$ which satisfies $l\left(\sigma_{n r}^{(n)}(u) v\right)=\psi_{U_{n r}}(u) l(v)$ for all $u \in U_{n r}$ and all vectors $v$ in the space of $\sigma_{n r}^{(n)}$. Given such a functional, we may consider the space of functions $W_{v}^{(n)}(h)=l\left(\sigma_{n r}^{(n)}(h) v\right)$.

Henceforth we shall assume that $\sigma_{n r}^{(n)}=\Theta_{n r}^{(n)}$ and denote the corresponding space of functions by $W_{n r}^{(n)}(h)$. Then, the following proposition is proved in [C] Theorem 1.2,

Proposition 1. The space of functionals $l$ defined as above on the representation $\Theta_{n r}^{(n)}$ is one dimensional. Moreover, for all $k \in K_{r}$ viewed as a subgroup of $G L_{r}^{\Delta}$, we have $W_{n r}^{(n)}(k h)=$ $W_{n r}^{(n)}(h)$. 
It is not hard to construct the space of functions $W_{n r}^{(n)}(h)$ explicitly on the space of $\Theta_{n r}^{(n)}$. Indeed, let $f \in I n d_{B_{n r}^{(n)}}^{G L_{n r}^{(n)}} \delta_{B_{n r}}^{\frac{n-1}{2 n}}$. Let $U_{n r}^{0}$ denote the subgroup of $U_{n r}$ which consists of all matrices $u$ as in (5) such that $X_{i, j} \in M a t_{r \times r}^{0}$ for all $i$ and $j$. Here $M a t_{r \times r}^{0}$ is the subgroup of $M a t_{r \times r}$ consisting of all matrices $X$ such that $X\left[l_{1}, l_{2}\right]=0$ for all $l_{1}<l_{2}$, where $X\left[l_{1}, l_{2}\right]$ denotes the $\left(l_{1}, l_{2}\right)$-th entry of $X$. Then

$$
W_{n r}^{(n)}(h)=\int_{U_{n r}^{0}} f\left(w_{J} w_{0} u h\right) \psi_{U_{n r}}(u) d u
$$

defines the space of functions which satisfies the required transformation properties, provided it is not identically zero. Here $w_{J}$ is the Weyl element $w_{J}=\operatorname{diag}\left(J_{n}, J_{n}, \ldots, J_{n}\right) \in G L_{n r}$ where $J_{n}$ is the longest Weyl element of $G L_{n}$. The Weyl element $w_{0}$ is defined as the element whose $(a+b n,(a-1) r+b+1)$ entry is one for all $1 \leq a \leq n$ and $0 \leq b \leq r-1$, and zero elsewhere. Matrix multiplication implies that $w_{J} w_{0}$ is the shortest Weyl element of $G L_{n r}$ with the property that for all $u \in U_{n r}^{0}$, we have that $w u w^{-1}$ is a lower unipotent matrix. By considering the function $W_{n r}^{(n)}(h)$ corresponding to the $K_{n r}$ fixed vector $f$ in the space of $\Theta_{n r}^{(n)}$, one can easily show that $W_{n r}^{(n)}(e) \neq 0$. This will follow from the computation which we will perform in the next Proposition.

Before doing that, it will be convenient to perform a simple computation which we will refer to several times. We will do it in the following subsection,

2.1. A local computation. Let $F$ denote a local field. Given a root $\alpha$ associated with the group $G L_{b}$, we will denote by $x_{\alpha}(l)$ the one dimensional unipotent subgroup of $G L_{b}$ associated with this root. Assume that $\alpha$ and $\beta$ are two roots such that $\alpha+\beta$ is also a root. Thus, we have $x_{\alpha}(z) x_{\beta}(l)=x_{\beta}(l) x_{\alpha}(z) x_{\alpha+\beta}(l z)$. Let $h(a)$ denote a one dimensional torus of $G L_{b}$ which satisfies the property $h(a)^{-1} x_{\alpha}(z) h(a)=x_{\alpha}\left(a^{-1} z\right)$ for all $a \in F^{*}$.

Let $f$ denote a function defined on $G L_{b}(F)$ which satisfies the property

$$
f\left(x_{\beta}\left(l_{1}\right) x_{\alpha+\beta}\left(l_{2}\right) g k\right)=\psi\left(-l_{2}\right) f(g)
$$

for all $k \in K_{b}$, where $K_{b}$ is the standard maximal compact subgroup of $G L_{b}$.

Our goal in this subsection is to compute the integral

$$
I=\int_{F^{2}} f\left(x_{\alpha}(z) x_{\beta}(l) h(a)\right) \psi(\epsilon l) d z d l
$$

Here $\epsilon=0,-1$. We have

Lemma 1. We have, $I=f\left(h(a) x_{\alpha}\left(-a^{-1} \epsilon\right)\right)=f\left(x_{\alpha}(-\epsilon) h(a)\right)$.

Proof. Since $f$ is right $K_{b}$ invariant, then

$$
I=\int_{F^{2}} \int_{|m| \leq 1} f\left(x_{\alpha}(z) x_{\beta}(l) h(a) x_{\alpha}(m)\right) \psi(\epsilon l) d m d z d l
$$

Conjugating $x_{\alpha}(m)$ to the left, and using the above assumptions on the commutation relations and properties (7), we obtain the integral $\int \psi(-l a m) d m$ as inner integration. Here $m$ is integrated over $|m| \leq 1$. Hence we may restrict the integration domain in integral $I$ to the domain $|l a| \leq 1$. 
The next step is to conjugate $x_{\beta}(l)$ to the left. Using the commutation relations and properties (7), we obtain

$$
I=\int_{F} f\left(x_{\alpha}(z) h(a)\right) \int_{|l a| \leq 1} \psi(z l+\epsilon l) d l d z
$$

Changing variables in $l$, we obtain

$$
I=|a|^{-1} \int_{\left|(z+\epsilon) a^{-1}\right| \leq 1} f\left(x_{\alpha}(z) h(a)\right) d z=|a|^{-1} \int_{\left|(z+\epsilon) a^{-1}\right| \leq 1} f\left(h(a) x_{\alpha}\left(a^{-1} z\right)\right) d z
$$

Writing $a^{-1} z=a^{-1} z+\epsilon a^{-1}-\epsilon a^{-1}$, we obtain

$$
I=|a|^{-1} f\left(h(a) x_{\alpha}\left(-a^{-1} \epsilon\right)\right) \int_{\left|(z+\epsilon) a^{-1}\right| \leq 1} d z
$$

from which the Lemma follows.

With the above notation we prove,

Corollary 1. We have

$$
\int_{F} f\left(x_{\alpha}(z)\right) d z=f(e)
$$

Proof. Since $f$ is right invariant under $K_{b}$, the above integral is equal to

$$
\int_{F} \int_{|m| \leq 1} f\left(x_{\alpha}(z) x_{\beta}(m)\right) d m d z
$$

Conjugating $x_{\beta}(m)$ to the left, we obtain from the left invariant properties of $f$, the integral $\int \psi(m z) d m$ as inner integration. Here $m$ is integrated over $|m| \leq 1$. The result follows.

2.2. On the generating function. In this subsection we will prove that the generating function can be expressed in term of the function $W_{n r}^{(n)}$.

Embed $g \in G L_{r}$ in $G L_{n r}$ as $g \mapsto g_{0}=\operatorname{diag}\left(g, I_{r}, \ldots, I_{r}\right)$. We have

Proposition 2. Let $W_{n r}^{(n)}(h)$ denote the function corresponding to the $K_{n r}$ fixed vector. Then, for $s^{\prime}=\frac{s}{n}-\frac{(n-2) r}{2}-\frac{1}{2 n}$ we have

$$
\widetilde{\Delta}_{s}(g)=\overline{W_{n r}^{(n)}\left(g_{0}\right)}|\operatorname{det} g|^{s^{\prime}}
$$

Proof. It follows from Proposition 1 that the function $W_{n r}^{(n)}\left(g_{0}\right)$ is $K_{r}$ bi-invariant. To prove the Proposition it is enough to show that

$$
\int_{G L_{r}} \omega_{\pi^{(n)}}(g) \overline{W_{n r}^{(n)}\left(g_{0}\right)}|\operatorname{det} g|^{s^{\prime}} d g=L\left(s, \pi^{(n)}\right)
$$

Notice that this will also imply that the function $W_{n r}^{(n)}(h)$ as defined in (6) is not identically zero on the space of the representation $\Theta_{n r}^{(n)}$. Using the identity $\omega_{\pi^{(n)}}(g)=\int_{K_{r}} f_{\pi^{(n)}}(k g) d k$ we 
may, after a change of variables, replace in (9) the function $\omega_{\pi^{(n)}}$ by $f_{\pi^{(n)}}$. Here $f_{\pi^{(n)}}$ is the unramified vector in the space of $\pi^{(n)}$. Performing the Iwasawa decomposition, the integral in equation (9) is equal to

$$
\int_{T_{r}} f_{\pi^{(n)}}(t) \int_{V_{r}} \overline{W_{n r}^{(n)}\left(v_{0} t_{0}\right)}|\operatorname{det} t|^{s^{\prime}} \delta_{B_{r}}(t)^{-1} d v_{0} d t
$$

Here $V_{r}$ is the maximal unipotent subgroup of $G L_{r}$ consisting of upper unipotent matrices. Also, $t=\operatorname{diag}\left(a_{1}, a_{2}, \ldots, a_{r}\right)$. Plug integral (6) in integral (10). Thus, we obtain the integral

$$
\int_{V_{r}} \int_{U_{n r}^{0}} \bar{f}\left(w_{J} w_{0} u v_{0} t_{0}\right) \psi_{U_{n r}}(u) d u d v_{0}
$$

as an inner integration to integral (10). Let $U_{n r}^{1}$ denote the subgroup of $U_{n r}^{0}$ consisting of all matrices such that $X_{1,2}[i, j]=0$ for all $i \neq j$. We claim that integral (11) is equal to

$$
\int_{U_{n r}^{1}} \bar{f}\left(w_{J} w_{0} u t_{0}\right) \psi_{U_{n r}}(u) d u
$$

We do this by using Lemma 1 several times. It is convenient to use the following notations. For all integers $1 \leq a, b \leq n r$ and all $m \in F$, let $x_{a, b}(m)=I_{n r}+m e_{a, b}$. Here $e_{a, b}$ is the matrix of size $n r$ which has a one in the $(a, b)$ entry, and zero elsewhere.

In integral (11) consider the integrations over the variables $X_{1,2}[r, r-1]$ and $v_{0}[r-1, r]$, where the last variable indicates the $(r-1, r)$ entry of $v_{0}$. In the notations of Subsection 2.1, let $x_{\alpha}(z)=x_{r, r-1}(z)$ where $z=X_{1,2}[r, r-1]$, and let $x_{\beta}(l)=x_{r-1, r}(l)$ where $l=$ $v_{0}[r-1, r]$. With these notations we have $x_{\alpha+\beta}(m)=x_{r-1,2 r-1}(m)$ and from the definition of the character $\psi_{U_{n r}}$ we have $\psi_{U_{n r}}\left(x_{\alpha+\beta}(m)\right) \neq 1$. Hence, all the conditions of Lemma 1 are satisfied with $\epsilon=0$ and $h(a)=h\left(a_{r}\right)=\operatorname{diag}\left(I_{r-1}, a_{r}, I_{n r-r}\right)$. From this we deduce that in the integral (11), we may restrict the domain of integration to the group $V_{r}$ with the condition that $v_{0}[r-1, r]=0$, and to the group $U_{n r}^{0}$ with the condition $X_{1,2}[r, r-1]=0$.

In general, we apply this process in the following order. Fix $r+1 \leq j \leq 2 r-1$. Then for all $j-r+1 \leq i \leq r$, set $x_{\alpha}(z)=x_{i, j}(z)$ with $z=X_{1,2}[i, j-r]$, and $x_{\beta}(l)=x_{j-r, i}(l)$ with $l=v_{0}[j-r, i]$. With these notations we have $x_{\alpha+\beta}(m)=x_{j-r, j}(m)$. Since $\psi_{U_{n r}}$ is not trivial on $x_{\alpha+\beta}(m)$, we can apply Lemma 1 with $\epsilon=0$. The end result of this repeated process is that integral (11) is equal to integral (12).

Conjugating by $w_{0}$, write $w_{0} U_{n r}^{1} w_{0}^{-1}=U_{n r}^{2} U_{n r}^{3}$, where the groups $U_{n r}^{2}$ and $U_{n r}^{3}$ are defined as follows. First, identify the group $U_{n r}^{2}$ with $r$ copies of the group $V_{n}$. Here $V_{n}$ is defined to be the group of all upper unipotent matrices of $G L_{n}$. The embedding of $U_{n r}^{2}$ inside $G L_{n r}$ is given by $\left(v_{n, 1}, v_{n, 2}, \ldots, v_{n, r}\right) \mapsto \operatorname{diag}\left(v_{n, 1}, v_{n, 2}, \ldots, v_{n, r}\right)$. Here $v_{n, i} \in V_{n}$. To define the group $U_{n r}^{3}$, consider the unipotent group generated by all matrices of the form

$$
\left(\begin{array}{ccccc}
I & & & & \\
Y_{2,1} & I & & & \\
Y_{3,1} & Y_{3,2} & I & & \\
\vdots & \vdots & \ddots & I & \\
Y_{r, 1} & Y_{r, 2} & \ldots & Y_{r, r-1} & I
\end{array}\right)
$$


Here $Y_{i, j}$ is in $\mathrm{Mat}_{n}$. Then the group $U_{n r}^{3}$ is generated by all matrices as in (13) which satisfies the conditions $Y_{i, j}\left[l_{1}, l_{2}\right]=Y_{i, j}[1,2]=0$ for all $l_{1} \geq l_{2}$.

For $v \in V_{n}$, let $\psi_{V_{n}}(v)$ denote the Whittaker character of the group $V_{n}$. This character is defined as follows. Given $v=(v[i, j]) \in V_{n}$, then

$$
\psi_{V_{n}}(v)=\psi(v[1,2]+v[2,3]+\cdots+v[n-1, n])
$$

Let $u_{2}=\operatorname{diag}\left(v_{n, 1}, v_{n, 2}, \ldots, v_{n, r}\right) \in U_{n r}^{2}$. Define the character $\psi_{U_{n r}^{2}}$ of $U_{n r}^{2}$ as $\psi_{U_{n r}^{2}}\left(u_{2}\right)=$ $\psi_{V_{n}}\left(v_{n, 1}\right) \psi_{V_{n}}\left(v_{n, 2}\right) \ldots \psi_{V_{n}}\left(v_{n, r}\right)$. Then, in the notations of $U_{n r}^{2} U_{n r}^{3}$ the character $\psi_{U_{n r}}$ transforms to the character $\psi_{U_{n r}^{2}}$ on the group $U_{n r}^{2}$, and is trivial on the group $U_{n r}^{3}$.

Thus, integral (12) is equal to

$$
\int_{U_{n r}^{3}} f_{W}\left(u_{3} w_{0} t_{0} w_{0}^{-1}\right) d u_{3}
$$

where

$$
f_{W}(h)=\int_{U_{n r}^{2}} \bar{f}\left(w_{J} u_{2} h\right) \psi_{U_{n r}^{2}}\left(u_{2}\right) d u_{2}
$$

We have $w_{0} t_{0} w_{0}^{-1}=\operatorname{diag}\left(A_{1}, A_{2}, \ldots, A_{r}\right)$ where $A_{i}=\operatorname{diag}\left(a_{i}, I_{n-1}\right)$. Conjugating the matrix $w_{0} t_{0} w_{0}^{-1}$ to the left in integral (15) we obtain the factor

$$
\alpha(t)=\left(\left|a_{2}\right|\left|a_{3}\right|^{2}\left|a_{4}\right|^{3} \ldots\left|a_{r}\right|^{r-1}\right)^{n-2}
$$

from the change of variables in $U_{n r}^{3}$. Thus, integral (15) is equal to

$$
\alpha(t) \int_{U_{n r}^{3}} f_{W}\left(w_{0} t_{0} w_{0}^{-1} u_{3}\right) d u_{3}
$$

We claim that integral (16) is equal to $\alpha(t) f_{W}\left(w_{0} t_{0} w_{0}^{-1}\right)$. This we will show by a repeated application of Corollary 1. Indeed, fix $2 \leq i \leq r$, where we first start with $i=r$, then $i=r-1$ and so on. Let $1 \leq k \leq i-1$. Assume that $l_{1}$ and $l_{2}$ are such that $Y_{i, k}\left[l_{1}, l_{2}\right]$ is a variable in the domain of integration in integral (16). In the notations of Subsection 2.1, let $x_{\alpha}(z)=$ $x_{(n-1) i+l_{1}, n(k-1)+l_{2}}(z)$ with $z=Y_{i, k}\left[l_{1}, l_{2}\right]$, and let $x_{\beta}(m)=x_{n(k-1)+l_{2},(n-1) i+l_{1}+1}(m)$. Then, $x_{\alpha+\beta}(l)=x_{(n-1) i+l_{1},(n-1) i+l_{1}+1}(l)$, and from the properties of $f_{W}$, we have $f_{W}\left(x_{\alpha+\beta}(l) g\right)=$ $\psi(-l) f_{W}(g)$. Applying Corollary 1 several times in the indicated order, the above claim follows.

Hence, integral (16) is equal to

$$
\alpha(t) f_{W}\left(w_{0} t_{0} w_{0}^{-1}\right)=\alpha(t) \delta_{B_{r}}^{\frac{n-1}{2}}(t) \prod_{i=1}^{r} W_{\Theta_{n}}\left(\begin{array}{cc}
a_{i} & \\
& I_{n-1}
\end{array}\right)
$$

Here $W_{\Theta_{n}}$ is the local Whittaker function associated to the Theta function of the group $G L_{n}^{(n)}$. Also, we have $\delta_{B_{r}}^{\frac{n-1}{2}}(t)=\delta_{P_{n, r}}^{\frac{n-1}{2 n}}\left(w_{0} t_{0} w_{0}^{-1}\right)$, where $P_{n, r}$ is the parabolic subgroup of $G L_{n r}$ whose Levi part is $G L_{n} \times G L_{n} \times \ldots \times G L_{n}$. It follows from [H] Propositions 5.1 and 5.3 that $W_{\Theta_{n}}\left(\begin{array}{cc}a_{i} & \\ & I_{n-1}\end{array}\right)=0$ unless $\left|a_{i}\right| \leq 1$ and $a_{i}=b_{i}^{n}$. In that case the 
value of the function is $\left|b_{i}\right|^{\frac{(n-1)^{2}}{2}}$. Notice that when $a_{i}=b_{i}^{n}$, then we have $f_{\pi^{(n)}}(t)=$ $\prod_{i=1}^{r} \chi_{i}^{n}\left(b_{i}\right) \delta_{B_{r}}^{n / 2}\left(\operatorname{diag}\left(1, \ldots, 1, b_{i}, 1, \ldots, 1\right)\right.$. Combing all this, integral (10) is equal to

$$
\prod_{i=1}^{r} \int_{\left|b_{i}\right| \leq 1} \chi_{i}^{n}\left(b_{i}\right)\left|b_{i}\right|^{n s^{\prime}+\frac{n(n-2)(r-1)}{2}+\frac{(n-1)^{2}}{2}} d b_{i}
$$

From this the Proposition follows.

\section{The Whittaker FUnCtional of the Generating FUnCTiON}

In this section we compute the Whittaker functional of the function $W_{n r}^{(n)}\left(g_{0}\right)$. Here the notations are as in Section 2, but we assume that $r<n$. We make this assumption to get a precise proof of Conjecture 1.2 in $[\mathrm{B}-\mathrm{F}$. The case when $r \geq n$ is similar and will be dealt with in the next section. Embed $g \in G L_{r}$ in $G L_{n}$ as $g \mapsto \operatorname{diag}\left(g, I_{n-r}\right)$. Let $g_{0}=\operatorname{diag}\left(g, I_{n}, \ldots, I_{n}\right) \in G L_{n r}$, where $I_{n}$ appears $r-1$ times.

Let $V_{r}$ denote the standard maximal unipotent subgroup of $G L_{r}$, and let $\psi_{V_{r}}^{-1}$ denote the Whittaker character of $V_{r}$. See equation (14) for the definition of $\psi_{V_{r}}$. Let $W_{\Theta_{n}}^{(n)}$ denote the Whittaker function of the Theta function defined on $G L_{n}^{(n)}$. Our goal is to prove

Theorem 2. Assume that $r<n$. With the above notations, for all $g \in G L_{r}^{(n)}$, we have

$$
\int_{V_{r}} W_{n r}^{(n)}\left(v_{0} g_{0}\right) \psi_{V_{r}}^{-1}(v) d v=W_{\Theta_{n}}^{(n)}\left(\begin{array}{ll}
g & \\
& I_{n-r}
\end{array}\right)|\operatorname{det} g|^{\frac{(n-1)(r-1)}{2}}
$$

Proof. We will consider the case when $r=n-1$. This is the hardest case. When $r<$ $n-1$ the computations are similar but simpler. Since we will use some of the notations introduced in the previous Sections, we will keep writing $r$ and $n$ even though we assume that $r=n-1$. By the Iwasawa decomposition, it is enough to prove identity (19) for $g=t=\operatorname{diag}\left(a_{1}, a_{2}, \ldots, a_{n-1}\right)$. Notice, that from the left invariant properties of $W_{n r}^{(n)}$ and $W_{\Theta_{n}}^{(n)}$, we may assume that $\left|a_{i}\right| \leq 1$ for all $1 \leq i \leq n-1$.

We start by plugging integral (6) into the left hand side of identity (19). Doing so, we obtain the integral

$$
\int_{V_{r}} \int_{U_{n r}^{0}} f\left(w_{J} w_{0} u v_{0} t_{0}\right) \psi_{U_{n r}}(u) \psi_{V_{r}}^{-1}(v) d u d v_{0}
$$

As in the proof of Proposition 2, we claim that integral (20) is equal to integral

$$
\int_{U_{n r}^{0}} f\left(w_{J} w_{0} u \delta_{0} t_{0}\right) \psi_{U_{n r}}(u) d u
$$

where $\delta_{0}=\prod_{i=2}^{n-1} x_{i, i-1}(1)$. Here, the definition of $U_{n r}^{0}$ and $x_{a, b}(m)$ are given before and after integral (12). To prove the above claim, we follow exactly the same steps as in the proof that integral (11) is equal to integral (12). The only difference, is that because of the character $\psi_{V_{r}}^{-1}$ in integral (20), then for the suitable variables in $V_{r}$, we need to use Lemma 11 with $\epsilon=-1$ and not with $\epsilon=0$ as in the proof of Proposition 2. This explains the element $\delta_{0}$. 
Next we proceed as in the proof of Proposition 2, Following the exact steps which showed that integral (12) is equal to integral (16), we deduce that integral (21) is equal to

$$
I_{1}=\alpha(t) \int_{U_{n r}^{3}} f_{W}\left(w_{0} t_{0} w_{0}^{-1} u_{3} \delta_{1}(t)\right) d u_{3}
$$

Here the group $U_{n r}^{3}$ and $\alpha(t)$ are defined before integral (16), and we remind the reader that we assume that $r=n-1$. Also, we have $\delta_{1}(t)=\prod_{i=2}^{n-1} x_{(i-1) n+1,(i-2) n+2}\left(a_{i}^{-1}\right)$. This element is obtained by conjugating $\delta_{0}$ by $w_{0}$ and $t_{0}$.

At this point, for all $2 \leq j \leq n-1$ we will introduce an integral which we denote by $I_{j}$. To do that we first fix some notations. Let $t_{j}=\operatorname{diag}\left(A_{j, j}, A_{j, j+1}, \ldots, A_{j, n-1}, I_{n}, \ldots, I_{n}\right)$ denote the torus element of $G L_{n r}$ where $A_{j, j}=\operatorname{diag}\left(a_{1}, \ldots, a_{j}, I_{n-j}\right)$ and for all $j+1 \leq$ $i \leq n-1$ we define $A_{j, i}=\operatorname{diag}\left(I_{j}, a_{i}, I_{n-j-1}\right)$. Notice that when $j=n-1$, we get $t_{j}=$ $\operatorname{diag}\left(A_{n-1, n-1}, I_{n}, \ldots, I_{n}\right)=t_{0}$. Next we define $\alpha_{j}(t)=\left|a_{j+1} a_{j+2}^{2} a_{j+3}^{3} \cdots a_{n-1}^{n-j-1}\right|^{n-2}$, where we set $\alpha_{n-1}(t)=1$. Finally, we define a set of subgroups $U_{n, j}$, and a set of characters $\psi_{U_{n, j}}$ defined on these groups. The definition is inductive, so we we start with $U_{n, 2}$. Consider the group $U_{n r}^{3}$ with $r=n-1$, as was defined right after equation (13). Let $U_{n, 2}$ denote the subgroup of $U_{n r}^{3}$ with the extra condition that $Y_{n-1, i}=0$ for all $1 \leq i \leq n-2$. Assuming we defined $U_{n, j-1}$ we define $U_{n, j}$ as the subgroup of $U_{n, j-1}$ consisting of matrices of the form (13) such that $Y_{n-j+1, i}=0$ for all $1 \leq i \leq n-j$, and also satisfies the condition $Y_{i, l}[b, j]=0$ for all $2 \leq i \leq n-j, 1 \leq l \leq i-1$ and $1 \leq b \leq n$. The character $\psi_{U_{n, j}}$ is defined as follows. For $u \in U_{n, j}$ written as in equation (13), we set $\psi_{U_{n, j}}(u)=\psi\left(\sum_{i=2}^{n-j} Y_{i, i-1}[j-1, j+1]\right)$.

With these notations, for all $2 \leq j \leq n-1$ we set

$$
I_{j}=\alpha_{j}(t) \int_{U_{n, j}} f_{W}\left(t_{j} u\right) \psi_{U_{n, j}}(u) d u
$$

We will prove that $I_{2}=I_{1}$, and that for all $2 \leq j \leq n-1$, we have $I_{j}=I_{j-1}$. This will complete the proof of the Theorem. Indeed, proving the above implies that the left hand side of equation (19) is equal to $I_{n-1}$. Since $\alpha_{n-1}(t)=1$, the group $U_{n, n-1}$ is the trivial group, and $t_{n-1}=t_{0}$, we deduce that $I_{n-1}=f_{W}\left(t_{0}\right)$. But as in equation (17) we obtain that $f_{W}\left(t_{0}\right)$ equals the right hand side of equation (19).

We prove that $I_{2}=I_{1}$. Since $\left|a_{i}\right| \leq 1$, we obtain the following Iwasawa decomposition $\delta_{1}(t)=\prod_{i=2}^{n-1} x_{(i-2) n+2,(i-1) n+1}\left(a_{i}\right) \prod_{i=2}^{n-1} h_{i}\left(a_{i}\right) k$. Here $k \in K_{n r}$, and we have $\prod_{i=2}^{n-1} h_{i}\left(a_{i}\right)=$ $\operatorname{diag}\left(B_{2,1}, B_{2,2}, \ldots, B_{2, n-1}\right)$. Here $B_{2,1}=\operatorname{diag}\left(1, a_{2}, I_{n-2}\right)$, for $2 \leq i \leq n-2$ we have $B_{2, i}=$ $\operatorname{diag}\left(a_{i}^{-1}, a_{i+1}, I_{n-2}\right)$ and $B_{2, n-1}=\operatorname{diag}\left(a_{n-1}^{-1}, I_{n-1}\right)$. Conjugating in $I_{1}$ the matrix $\delta_{1}(t) k^{-1}$ to left, and using the left invariant properties of $f_{W}$, we obtain by matrix multiplication

$$
I_{1}=\alpha_{2}(t) \int_{U_{n r}^{3}} f_{W}\left(t_{2} u_{3}\right) \psi_{U_{n r}^{3}}\left(u_{3}\right) d u_{3}
$$

Here we use the fact that $w_{0} t_{0} w_{0}^{-1} \prod_{i=2}^{n-1} h_{i}\left(a_{i}\right)=t_{2}$. The factor of $\left|a_{2} a_{3} \ldots a_{n-1}\right|^{-(n-2)}$ is obtained from a change of variables when we conjugate the torus $\prod_{i=2}^{n-1} h_{i}\left(a_{i}\right)$ across $U_{n r}^{3}$. The product of this factor by $\alpha_{1}(t)$ is equal to $\alpha_{2}(t)$. The character $\psi_{U_{n r}^{3}}$ is defined as follows. For $u_{3} \in U_{n r}^{3}$ define $\psi_{U_{n r}^{3}}\left(u_{3}\right)=\psi\left(\sum_{i=2}^{n-1} Y_{i, i-1}[1,3]\right)$. To complete the proof that $I_{2}=I_{1}$, we need to show that we can restrict the support of integration from $U_{n r}^{3}$ to $U_{n, 2}$. 
In other words, we need to show that for all $1 \leq i \leq n-2$, the integration over all variables in $Y_{n-1, i}$ is in $K_{n r}$. This is done as in the proof of Proposition 2 while showing that integral (16) reduces to the left hand side of identity (17). Indeed, from the definition of the torus $t_{2}$, given a variable $Y_{n-1, i}\left[l_{1}, l_{2}\right]$, we can find a one dimensional unipotent subgroup $x_{\beta}(m)$ so that we can apply Corollary 1. Thus $I_{2}=I_{1}$.

The next step is to prove that $I_{j}=I_{j-1}$. The first step is to prove that we can integrate over a smaller unipotent group. Let $U_{n, j-1,1}$ denote the subgroup of $U_{n, j-1}$ consisting of all matrices which also satisfies $Y_{i, l}[b, j]=0$ for all $3 \leq i \leq n-j+1,1 \leq l \leq i-2$ and $1 \leq b \leq j-1$. To show that we can reduce the domain of integration from $U_{n, j-1}$ to $U_{n, j-1,1}$ we apply Corollary 1. In the notations of this Corollary, let $x_{\alpha}(z)=x_{n(i-1)+b, n(l-1)+j}(z)$ with $z=Y_{i, l}[b, j]$, and let $x_{\beta}(m)=x_{n l+j-2, n(i-1)+b}(m)$. Notice that in this case the root $\alpha+\beta$ corresponds to the one dimensional unipotent subgroup $x_{n l+j-2, n(l-1)+j}(c)$, which is a subgroup of $U_{n, j-1,1}$. Moreover, the character $\psi_{U_{n, j-1}}$ is not trivial on this subgroup. Hence, the conditions of the Corollary 1 are satisfied. We mention that the order for which we apply this Corollary is important. We first vary $3 \leq i \leq n-j+1$ and fix $l=1$. Then we repeat the same process with $l=2$ and so on.

The second step is to show that $I_{j-1}$ is equal to

$$
\alpha_{j-1}(t) \int_{U_{n, j-1,2}} f_{W}\left(t_{j-1} u \delta_{j-1}(t)\right) \psi_{U_{n, j-1}}(u) d u
$$

Here $U_{n, j-1,2}$ is the subgroup of $U_{n, j-1,1}$ which satisfies the condition that $Y_{i, i-1}[j-1, j]$ for all $2 \leq i \leq n-j+1$. The matrix $\delta_{j-1}(t)=\prod_{i=2}^{n-j+1} x_{(i-1) n+j-1, n(i-2)+j}\left(a_{j+i-2}^{-1}\right)$. To derive integral (23) we apply Lemma 1 with $x_{\alpha}(z)=x_{n(i-1)+j-1, n(i-2)+j}(z)$ with $z=Y_{i, i-1}[j-1, j]$ and $x_{\beta}(l)=x_{(i-1) n+j-2,(i-1) n+j-1}(l)$. The next step is to perform an Iwasawa decomposition for $\delta_{j-1}(t)$ in integral (23) . This is done as with $\delta_{1}(t)$ and we obtain

$$
\delta_{j-1}(t)=\prod_{i=2}^{n-j+1} x_{n(i-2)+j,(i-1) n+j-1}\left(a_{j+i-2}\right) \prod_{i=2}^{n-j+1} h_{i}^{\prime}\left(a_{j+i-2}\right) k
$$

where $k \in K_{n r}$. Here $\prod_{i=2}^{n-j+1} h_{i}^{\prime}\left(a_{j+i-2}\right)=\operatorname{diag}\left(B_{j-1,1}, B_{j-1,2}, \ldots, B_{j-1, n-j+1}, I_{n}, \ldots, I_{n}\right)$, where $B_{j-1,1}=\operatorname{diag}\left(I_{j-1}, a_{j}, I_{n-j}\right), B_{j-1, i}=\operatorname{diag}\left(I_{j-2}, a_{j+i-2}^{-1}, a_{j+i-1}, I_{n-j}\right)$ for $2 \leq i \leq n-j$, and $B_{j-1, n-j+1}=\operatorname{diag}\left(I_{j-2}, a_{n-1}^{-1}, I_{n-j+1}\right)$. Plugging this into integral (23) and conjugate the matrix $\delta_{j-1}(t) k^{-1}$ to the left, we obtain

$$
\alpha_{j}(t) \int_{U_{n, j-1,2}} f_{W}\left(t_{j} u\right) \psi_{U_{n, j}}(u) d u
$$

Here, we obtain the factor of $\left|a_{j} a_{j+1} \ldots a_{n-1}\right|^{-(n-2)}$ from the conjugation of the toral part of $\delta_{j-1}(t) k^{-1}$ across $U_{n, j-1,2}$. This combined with $\alpha_{j-1}(t)$ gives the factor $\alpha_{j}(t)$ in integral (24). Notice also that the conjugation by the unipotent part of $\delta_{j-1}(t) k^{-1}$ changes the additive character to $\psi_{U_{n, j}}$. This is well defined. Indeed, notice that $U_{n, j}$ is a subgroup $U_{n, j-1,2}$ and we can view $\psi_{U_{n, j}}$ as a character of $U_{n, j-1,2}$ by extending it trivially. Finally, we have the identity $t_{j-1} \prod_{i=2}^{n-j+1} h_{i}^{\prime}\left(a_{j+i-2}\right)=t_{j}$. To show that integral (24) equals $I_{j}$, we need to show that we may restrict the domain of integration from $U_{n, j-1,2}$ to $U_{n, j}$. We do so using Corollary 1. Indeed, the group $U_{n, j}$ is the subgroup of $U_{n, j-1,2}$ obtained by setting $Y_{n-j+1, l}=0$ for 
all $1 \leq l \leq n-j$ and $Y_{i, i-1}[b, j]=0$ for all $2 \leq i \leq n-j$ and $1 \leq b \leq j-2$. To show that we may restrict the integration over $U_{n, j-1,2}$ to the subgroup obtained by setting $Y_{n-j+1, l}=0$, we argue in a similar way as in the reduction from the group $U_{n r}^{3}$ to $U_{n, 2}$ as was done right after integral (22). Then, finally to obtain the group $U_{n, j}$ we use Corollary 1 with $x_{\alpha}(z)=x_{n(i-1)+b, n(i-2)+j}(z)$ where $z=Y_{i, i-1}[b, j]$ and $x_{n(i-2)+j, n(i-1)+b+1}(m)$. Here $2 \leq i \leq n-j$ and $1 \leq b \leq j-2$.

\section{THE CASE WHEN $r \geq n$}

As mentioned in the introduction, the authors of $[\mathrm{B}-\mathrm{F}]$ were well aware that the situation when $r \geq n$ is similar. Since they do not specify this case explicitly, we briefly mention the global constructions and show how a similar result to Theorem 2 holds in this case.

Assume first that $r>n$. Let $\pi^{(n)}$ denote a cuspidal representation of the group $G L_{r}^{(n)}(\mathbf{A})$. Let $\Theta_{n}^{(n)}$ denote the Theta representation of the group $G L_{n}^{(n)}(\mathbf{A})$. Then we consider the global integral (11) introduced in the introduction. The group $V_{r, n}$ is defined as follows. Recall that $V_{r}$ is the standard maximal unipotent subgroup of $G L_{r}$. Then, $V_{r, n}$ is the subgroup of $V_{r}$ consisting of all matrices $v=\left(v_{i, j}\right) \in V_{r}$ such that $v_{i, j}=0$ for all $2 \leq j \leq n+1$. The character $\psi_{V_{r, n}}$ is defined by $\psi_{V_{r, n}}(v)=\psi\left(v_{n+1, n+2}+v_{n+2, n+3}+\cdots+v_{r-1, r}\right)$. It follows from the cuspidality of $\phi$ that integral (11) converges for all $s$. A similar unfolding as in [B-F] Section 2, implies that for $\operatorname{Re}(s)$ large, integral (11) is equal to integral (2).

Next we consider the case when $r=n$. In this case the global integral is given by

$$
\int_{Z_{r}(\mathbf{A}) G L_{r}(F) \backslash G L_{r}(\mathbf{A})} \phi(g) \overline{\theta(g)} E(g, s) d g
$$

Here $Z_{r}$ is the subgroup of $Z$, the center of $G L_{r}$, which consists of scalar matrices which are $r$ powers. For simplicity we assume that all representations have a trivial central character. Also, $E(g, s)$ is the Eisenstein series defined on the group $G L_{r}(\mathbf{A})$ and is associated with the induced representation $\operatorname{Ind}_{P(\mathbf{A})}^{G L_{r}(\mathbf{A})} \delta_{P}^{s}$. Here $P$ is the maximal parabolic subgroup of $G L_{r}$ whose Levi part is $G L_{r-1} \times G L_{1}$. Unfolding this integral, by first unfolding the Eisenstein series, we obtain for $\operatorname{Re}(s)$ large, that integral (25) is equal to

$$
\int_{Z_{r}(\mathbf{A}) V_{r}(\mathbf{A}) \backslash G L_{r}(\mathbf{A})} W_{\phi}(g) \overline{W_{\theta}(g)} f(g, s) d g
$$

Here $f(g, s)$ is a section in the above induced representation.

Next we study the local unramified computation corresponding to the integrals (2) and (26). Since the Whittaker coefficient of the representation $\pi^{(n)}$ is not factorizable, it is not clear that these integrals are Eulerian. However, as explained in [B-F], if we can prove similar results to Proposition 2 and to Theorem 2, the so call New way method would imply that these integrals are indeed factorizable. As for Proposition 2, it is clear that it holds for all values of $r$ and $n$.

As for Theorem 2, this is not the case for all matrices $g \in G L_{r}^{(n)}$. Assuming $r \geq n$, denote by $T_{0}$ the subgroup of $G L_{r}$ which consists of all diagonal matrices $t=\operatorname{diag}\left(a_{1}, a_{2}, \ldots, a_{r}\right)$ such that $\left|a_{i}\right| \leq 1$ for $1 \leq i \leq n$ and $\left|a_{i}\right|=1$ for all $n+1 \leq i \leq r$. Let $T_{0}^{(n)}$ denote the inverse image of $T_{0}$ inside $G L_{r}^{(n)}$. Let $G L_{r, 0}^{(n)}$ denote all elements $g \in G L_{r}^{(n)}$ which can be written 
as $g=v t k$ where $v \in V_{r}, t \in T_{0}^{(n)}$ and $k \in K_{r}$. Here $K_{r}$ is the standard maximal compact subgroup of $G L_{r}$. With these notations we have

Theorem 3. Assume that $r \geq n$. Then, for all $g \in G L_{r, 0}^{(n)}$, we have

$$
\int_{V_{r}} W_{n r}^{(n)}\left(v_{0} g_{0}\right) \psi_{V_{r}}^{-1}(v) d v=W_{\Theta_{n}}^{(n)}(g)|\operatorname{det} g|^{\frac{(n-1)(r-1)}{2}}
$$

Proof. The proof of this Theorem is the same as the proof of Theorem 2, and so we will only indicate the end result. Using the Iwasawa decomposition, we may assume that $g_{0}=t_{0}=$ $\operatorname{diag}\left(t, I_{r}, \ldots, I_{r}\right)$ where $t=\left(a_{1}, \ldots, a_{r}\right)$.

Defining similar integrals $I_{j}$ as in Theorem 2, we prove that the left hand side of integral (27) is equal to $f_{W}\left(t_{0}^{\prime}\right)$ where $t_{0}^{\prime}=\operatorname{diag}\left(A_{1}, A_{2}, \ldots, A_{r-n+1}, I_{n}, \ldots, I_{n}\right)$. Here $A_{1}=$ $\operatorname{diag}\left(a_{1}, \ldots, a_{n}\right)$ and for all $2 \leq i \leq r-n+1$ we have $A_{i}=\operatorname{diag}\left(I_{n-1}, a_{n+i-1}\right)$. Applying the factorization of equation (17), we obtain the identity

$$
f_{W}\left(t_{0}^{\prime}\right)=\delta_{P_{r, n}}^{\frac{n-1}{2 n}}\left(t_{0}^{\prime}\right) \prod_{i=1}^{r-n+1} W_{\Theta_{n}}^{(n)}\left(A_{i}\right)
$$

From the properties of the Whittaker function, we deduce that for all $2 \leq i \leq r-n+1$ we have $W_{\Theta_{n}}^{(n)}\left(A_{i}\right)=0$ unless $\left|a_{n+i-1}\right|=1$. From this the Theorem follows.

Notice that this Theorem is enough to prove that the corresponding local integrals of integrals (2) and (26), are Eulerian. Indeed, the local version of integral (2) is given by

$$
\int_{V_{r} \backslash G L_{r}} W_{\phi}\left(\begin{array}{ll}
g & \\
& I_{r-n}
\end{array}\right) \overline{W_{\theta}(g)}|\operatorname{det} g|^{s-\frac{r-n}{2}} d g
$$

Here $\phi$ is a vector in the local component of $\pi_{\nu}^{(n)}$ where $\nu$ is a place where all data is unramified. Similarly for $\theta$. Also, $W_{\phi}$ is any local Whittaker functional defined on the representation $\pi_{\nu}^{(n)}$. Similarly $W_{\theta}$ is the Whittaker functional defined on the space of $\Theta_{n, \nu}^{(n)}$. It is known that for the representation $\Theta_{n, \nu}^{(n)}$ this Whittaker functional is unique ( see [K-P]). However, this need not be the case for the representation $\pi_{\nu}^{(n)}$.

Applying the Iwasawa decomposition to the quotient $V_{r} \backslash G L_{r}$, the domain of integration in integral (28) is reduced to the torus $T_{r}$ of $G L_{r}$. However, because of the Whittaker functional

properties, $W_{\phi}\left(\begin{array}{cc}t & \\ & I_{r-n}\end{array}\right)$ is zero unless $t \in T_{0}$. Hence, we can apply Theorem 3 to deduce that integral (2) is indeed Eulerian.

A similar argument applies to integral (26). Indeed, using the properties of the Whittaker function, we can choose representatives for the quotient $Z_{r} \backslash T_{r}$ to be in the group $T_{0}$. Hence, once again we can apply Theorem 3 .

\section{REFERENCES}

[B-F] Bump, Daniel; Friedberg, Solomon Metaplectic generating functions and Shimura integrals. Automorphic forms, automorphic representations, and arithmetic (Fort Worth, TX, 1996), 117, Proc. Sympos. Pure Math., 66, Part 2, Amer. Math. Soc., Providence, RI, 1999. 
[C] Cai, Yuanqing Fourier Coefficients for Theta Representations on Covers of General Linear Groups, arXiv:1602.06614.

[H] Hoffstein, Jeff Eisenstein series and theta functions on the metaplectic group. Theta functions: from the classical to the modern, 65104, CRM Proc. Lecture Notes, 1, Amer. Math. Soc., Providence, RI, 1993.

[K-P] Kazhdan, D. A.; Patterson, S. J. Metaplectic forms. Inst. Hautes tudes Sci. Publ. Math. No. 59 (1984), 35142.

[PS-R] Piatetski-Shapiro, I.; Rallis, S. A new way to get Euler products. J. Reine Angew. Math. 392 (1988), 110124.

Ginzburg: School of Mathematical Sciences, Tel Aviv University, Ramat Aviv, Tel Aviv 6997801, ISRAEL

E-mail address: ginzburg@post.tau.ac.il 